\title{
Solutions Form for Some Rational Systems of Difference Equations
}

\author{
H. El-Metwally ${ }^{1,2}$ \\ ${ }^{1}$ Department of Mathematics, Rabigh College of Science and Art, King Abdulaziz University, P.O. Box 344, Rabigh 21911, Saudi Arabia \\ ${ }^{2}$ Department of Mathematics, Faculty of Science, Mansoura University, Mansoura 35516, Egypt
}

Correspondence should be addressed to H. El-Metwally; helmetwally2001@yahoo.com

Received 13 February 2013; Accepted 8 April 2013

Academic Editor: Ibrahim Yalcinkaya

Copyright (C) $2013 \mathrm{H}$. El-Metwally. This is an open access article distributed under the Creative Commons Attribution License, which permits unrestricted use, distribution, and reproduction in any medium, provided the original work is properly cited.

We deal with the form of the solutions for the following systems of rational difference equations $x_{n+1}=\left(x_{n-1} y_{n} /\left( \pm x_{n-1} \pm y_{n-2}\right)\right)$, $y_{n+1}=\left(x_{n} y_{n-1} /\left( \pm y_{n-1} \pm x_{n-2}\right)\right)$, with nonzero real numbers initial conditions. Also we investigate some properties of the obtained solutions and present some numerical examples.

\section{Introduction}

Our aim in this paper is to find the solutions form for the following systems of rational difference equations:

$$
x_{n+1}=\frac{x_{n-1} y_{n}}{ \pm x_{n-1} \pm y_{n-2}}, \quad y_{n+1}=\frac{x_{n} y_{n-1}}{ \pm y_{n-1} \pm x_{n-2}}, \quad n=0,1, \ldots,
$$

with nonzero real numbers initial conditions and then investigate the obtained solutions.

Difference equations appear naturally as discrete analogues and as numerical solutions of differential and delay differential equations having applications in biology, ecology, economy, physics, and so on. So, recently there has been an increasing interest in the study of qualitative analysis of scalar rational difference equations and systems of rational difference equations. Although difference equations are very simple in form, it is extremely difficult to understand thoroughly the behaviors of their solutions. See [1-7] and the references cited therein.

The periodicity of the positive solutions for the following system of rational difference equations

$$
x_{n+1}=\frac{m}{y_{n}}, \quad y_{n+1}=\frac{p y_{n}}{x_{n-1} y_{n-1}}
$$

was studied by Cinar et al. [8].
Özban [9] has studied the positive solutions for the following system:

$$
x_{n+1}=\frac{a}{y_{n-3}}, \quad y_{n+1}=\frac{b y_{n-3}}{x_{n-q} y_{n-q}} .
$$

The behavior of the positive solutions for the following system

$$
x_{n+1}=\frac{x_{n-1}}{1+x_{n-1} y_{n}}, \quad y_{n+1}=\frac{y_{n-1}}{1+y_{n-1} x_{n}}
$$

has been studied by Kurbanlı et al. [10].

Touafek and Elsayed [11] studied the periodicity and gave the form of the solutions for the following systems:

$$
x_{n+1}=\frac{y_{n}}{x_{n-1}\left( \pm 1 \pm y_{n}\right)}, \quad y_{n+1}=\frac{x_{n}}{y_{n-1}\left( \pm 1 \pm x_{n}\right)} .
$$

Yalcinkaya [12] investigated the sufficient condition for the global asymptotic stability for the following system of difference equations:

$$
z_{n+1}=\frac{t_{n} z_{n-1}+a}{t_{n}+z_{n-1}}, \quad t_{n+1}=\frac{z_{n} t_{n-1}+a}{z_{n}+t_{n-1}} .
$$

Yang [13] investigated the positive solutions for the system

$$
x_{n}=A+\frac{y_{n-1}}{x_{n-p} y_{n-q}}, \quad y_{n}=A+\frac{x_{n-1}}{x_{n-r} y_{n-s}} .
$$


Clark et al. $[14,15]$ investigate the global asymptotic stability of the following difference equations:

$$
x_{n+1}=\frac{x_{n}}{a+c y_{n}}, \quad y_{n+1}=\frac{y_{n}}{b+d x_{n}} \text {. }
$$

Camouzis and Papaschinopoulos [16] studied the global asymptotic behavior of the positive solutions of the system of rational difference equations as follows:

$$
x_{n+1}=1+\frac{x_{n}}{y_{n-m}}, \quad y_{n+1}=\frac{y_{n}}{x_{n-m}} .
$$

2. On the System: $x_{n+1}=x_{n-1} y_{n} /\left(x_{n-1}+y_{n-2}\right)$,

$$
y_{n+1}=x_{n} y_{n-1} /\left(y_{n-1}+x_{n-2}\right)
$$

In this section, we study the existence of analytical forms of the solutions for the following system of difference equations:

$$
x_{n+1}=\frac{x_{n-1} y_{n}}{x_{n-1}+y_{n-2}}, \quad y_{n+1}=\frac{x_{n} y_{n-1}}{y_{n-1}+x_{n-2}}, \quad n=0,1, \ldots,
$$

with nonzero real initials conditions $x_{-2}, x_{-1}, x_{0}, y_{-2}, y_{-1}$, and $y_{0}$.

In the sequel we assume that $\prod_{i=0}^{-1} A_{i} B_{i}=1$, for any real numbers $A_{i}$ and $B_{i}$.

Theorem 1. Suppose that $\left\{x_{n}, y_{n}\right\}$ is a solution for system (10), then for $n=0,1,2, \ldots$, one obtains

$$
\begin{gathered}
x_{2 n-2}=\frac{c(a e)^{n}}{\prod_{i=0}^{n-1}(i a+e)(i e+c)}, \\
x_{2 n-1}=\frac{b(b d)^{n}}{\prod_{i=0}^{n-1}(i d+b)((i+1) b+f)}, \\
y_{2 n-2}=\frac{f(b d)^{n}}{\prod_{i=0}^{n-1}(i d+b)(i b+f)}, \\
y_{2 n-1}=\frac{e(a e)^{n}}{\prod_{i=0}^{n-1}(i a+e)((i+1) e+c)},
\end{gathered}
$$

where $c=x_{-2}, b=x_{-1}, a=x_{0}, f=y_{-2}, e=y_{-1}$, and $d=y_{0}$.

Proof. For $n=0$ the result holds. Now suppose that $n>0$ and that our assumption holds for $n-1$. That is,

$$
\begin{gathered}
x_{2 n-5}=\frac{b(b d)^{n-2}}{\prod_{i=0}^{n-3}(i d+b)((i+1) b+f)}, \\
x_{2 n-4}=\frac{c(a e)^{n-1}}{\prod_{i=0}^{n-2}(i a+e)(i e+c)}, \\
x_{2 n-3}=\frac{b(b d)^{n-1}}{\prod_{i=0}^{n-2}(i d+b)((i+1) b+f)}, \\
y_{2 n-5}=\frac{e(a e)^{n-2}}{\prod_{i=0}^{n-3}(i a+e)((i+1) e+c)},
\end{gathered}
$$

$$
\begin{gathered}
y_{2 n-4}=\frac{f(b d)^{n-1}}{\prod_{i=0}^{n-2}(i d+b)(i b+f)}, \\
y_{2 n-3}=\frac{e(a e)^{n-1}}{\prod_{i=0}^{n-2}(i a+e)((i+1) e+c)} .
\end{gathered}
$$

Now, it follows from system (10) that

$$
\begin{aligned}
& x_{2 n-2}=\frac{x_{2 n-4} y_{2 n-3}}{x_{2 n-4}+y_{2 n-5}} \\
& =\left(\frac{c(a e)^{n-1}}{\prod_{i=0}^{n-2}(i a+e)(i e+c)}\right) \\
& \times\left(\frac{e(a e)^{n-1}}{\prod_{i=0}^{n-2}(i a+e)((i+1) e+c)}\right) \\
& \times\left(\left(\frac{c(a e)^{n-1}}{\prod_{i=0}^{n-2}(i a+e)(i e+c)}\right)\right. \\
& \left.+\left(\frac{e(a e)^{n-2}}{\prod_{i=0}^{n-3}(i a+e)((i+1) e+c)}\right)\right)^{-1} \\
& =\left(\frac{c(a e)^{n}}{\prod_{i=0}^{n-2}(i a+e)(i e+c)}\right) \\
& \times\left(\left(\frac{c a \prod_{i=0}^{n-2}(i a+e)((i+1) e+c)}{\prod_{i=0}^{n-2}(i a+e)(i e+c)}\right)\right. \\
& \left.+\left(\frac{\prod_{i=0}^{n-2}(i a+e)((i+1) e+c)}{\prod_{i=0}^{n-3}(i a+e)((i+1) e+c)}\right)\right)^{-1} \\
& =\left(\frac{c(a e)^{n}}{\prod_{i=0}^{n-2}(i a+e)(i e+c)}\right) \\
& \times((a((n-1) e+c)) \\
& +(((n-2) a+e)((n-1) e+c)))^{-1} \\
& =\frac{\left(c(a e)^{n} / \prod_{i=0}^{n-2}(i a+e)(i e+c)\right)}{((n-1) e+c)(a+((n-2) a+e))} \\
& =\frac{\left(c(a e)^{n} / \prod_{i=0}^{n-2}(i a+e)(i e+c)\right)}{((n-1) e+c)((n-1) a+e)} \\
& =\frac{c(a e)^{n}}{\prod_{i=0}^{n-1}(i a+e)(i e+c)}, \\
& y_{2 n-2}=\frac{y_{2 n-4} x_{2 n-3}}{y_{2 n-4}+x_{2 n-5}} \\
& =\left(\frac{f(b d)^{n-1}}{\prod_{i=0}^{n-2}(i d+b)(i b+f)}\right) \\
& \times\left(\frac{b(b d)^{n-1}}{\prod_{i=0}^{n-2}(i d+b)((i+1) b+f)}\right)
\end{aligned}
$$




$$
\begin{aligned}
& \times\left(\left(\frac{f(b d)^{n-1}}{\prod_{i=0}^{n-2}(i d+b)(i b+f)}\right)\right. \\
& \left.\quad+\left(\frac{b(b d)^{n-2}}{\prod_{i=0}^{n-3}(i d+b)((i+1) b+f)}\right)\right)^{-1} \\
& =\left(\frac{f(b d)^{n}}{\prod_{i=0}^{n-2}(i d+b)(i b+f)}\right) \\
& \times(d((n-1) b+f) \\
& \quad+((n-2) d+b)((n-1) b+f))^{-1} \\
& =\frac{\left(f(b d)^{n} / \prod_{i=0}^{n-2}(i d+b)(i b+f)\right)}{((n-1) b+f)[d+((n-2) d+b)]} \\
& =\frac{f(b d)^{n}}{\prod_{i=0}^{n-1}(i d+b)(i b+f)}
\end{aligned}
$$

Also, from system (10), we see that

$$
\begin{aligned}
& x_{2 n-1}=\frac{x_{2 n-3} y_{2 n-2}}{x_{2 n-3}+y_{2 n-4}} \\
& =\left(\frac{b(b d)^{n-1}}{\prod_{i=0}^{n-2}(i d+b)((i+1) b+f)}\right) \\
& \times\left(\frac{f(b d)^{n}}{\prod_{i=0}^{n-1}(i d+b)(i b+f)}\right) \\
& \times\left(\left(\frac{b(b d)^{n-1}}{\prod_{i=0}^{n-2}(i d+b)((i+1) b+f)}\right)\right. \\
& \left.+\left(\frac{f(b d)^{n-1}}{\prod_{i=0}^{n-2}(i d+b)(i b+f)}\right)\right)^{-1} \\
& =\left(\frac{f b d b(b d)^{n-1}}{\prod_{i=0}^{n-2}(i d+b)((i+1) b+f)}\right) \\
& \times(b f((n-1) d+b) \\
& +f((n-1) d+b)((n-1) b+f))^{-1} \\
& =\frac{\left(b(b d)^{n} / \prod_{i=0}^{n-2}(i d+b)((i+1) b+f)\right)}{((n-1) d+b)[b+(n-1) b+f]} \\
& =\frac{b(b d)^{n}}{\prod_{i=0}^{n-1}(i d+b)((i+1) b+f)}, \\
& y_{2 n-1}=\frac{y_{2 n-3} x_{2 n-2}}{y_{2 n-3}+x_{2 n-4}} \\
& =\left(\frac{e(a e)^{n}}{\prod_{i=0}^{n-2}(i a+e)((i+1) e+c)}\right)
\end{aligned}
$$

$$
\begin{aligned}
& \times(e((n-1) a+e)+((n-1) a+e)((n-1) e+c))^{-1} \\
= & \frac{e(a e)^{n}}{\prod_{i=0}^{n-1}(i a+e)((i+1) e+c)} .
\end{aligned}
$$

The proof is complete.

Lemma 2. Every positive solution for system (10) is bounded, and $\lim _{n \rightarrow \infty} x_{n}=\lim _{n \rightarrow \infty} y_{n}=0$.

Proof. It follows from system (10) that

$$
\begin{gathered}
x_{n+1}=\frac{x_{n-1} y_{n}}{x_{n-1}+y_{n-2}}<\frac{x_{n-1} y_{n}}{x_{n-1}}=y_{n}, \\
y_{n+1}=\frac{x_{n} y_{n-1}}{y_{n-1}+x_{n-2}}<\frac{x_{n} y_{n-1}}{y_{n-1}}=x_{n},
\end{gathered}
$$

for $n$ large, we see that

$$
x_{n+1}<y_{n}<x_{n-1}, \quad y_{n+1}<x_{n}<y_{n-1} .
$$

Then the subsequences $\left\{x_{2 n-1}\right\}_{n=0}^{\infty},\left\{x_{2 n}\right\}_{n=0}^{\infty},\left\{y_{2 n-1}\right\}_{n=0}^{\infty}$, and $\left\{y_{2 n}\right\}_{n=0}^{\infty}$ are decreasing and so are bounded from above by $M, M, N$, and $N$, respectively, where $M=\max \left\{x_{-1}, x_{0}\right\}$ and $N=\max \left\{y_{-1}, y_{0}\right\}$.

The proofs of the following theorems are similar to that of Theorem 1 and will be omitted.

Theorem 3. Assume that $\left\{x_{n}, y_{n}\right\}$ is a solution for the system

$$
x_{n+1}=\frac{x_{n-1} y_{n}}{x_{n-1}+y_{n-2}}, \quad y_{n+1}=\frac{x_{n} y_{n-1}}{x_{n-2}-y_{n-1}} .
$$

Then for $n=0,1,2, \ldots$,

$$
\begin{gathered}
x_{2 n-2}=\frac{c(a e)^{n}}{\prod_{i=0}^{n-1}(e+i a)(c-i e)}, \\
x_{2 n-1}=\frac{b(b d)^{n}}{\prod_{i=0}^{n-1}(b-i d)(f+(i+1) b)}, \\
y_{2 n-2}=\frac{f(b d)^{n}}{\prod_{i=0}^{n-1}(b-i d)(f+i b)}, \\
y_{2 n-1}=\frac{e(a e)^{n}}{\prod_{i=0}^{n-1}(e+i a)(c-(i+1) e)} .
\end{gathered}
$$

Theorem 4. The solutions form for the following system:

$$
x_{n+1}=\frac{x_{n-1} y_{n}}{y_{n-2}-x_{n-1}}, \quad y_{n+1}=\frac{x_{n} y_{n-1}}{y_{n-1}+x_{n-2}}
$$


are given by the following formulas:

$$
\begin{gathered}
x_{2 n-2}=\frac{c(a e)^{n}}{\prod_{i=0}^{n-1}(e-i a)(c+i e)}, \\
x_{2 n-1}=\frac{b(b d)^{n}}{\prod_{i=0}^{n-1}(b+i d)(f-(i+1) b)}, \\
y_{2 n-2}=\frac{f(b d)^{n}}{\prod_{i=0}^{n-1}(b+i d)(f-i b)}, \\
y_{2 n-1}=\frac{e(a e)^{n}}{\prod_{i=0}^{n-1}(e-i a)(c+(i+1) e)} .
\end{gathered}
$$

Theorem 5. Let $\left\{x_{n}, y_{n}\right\}$ be a solution for the following system of difference equations

$$
x_{n+1}=\frac{x_{n-1} y_{n}}{y_{n-2}-x_{n-1}}, \quad y_{n+1}=\frac{x_{n} y_{n-1}}{x_{n-2}-y_{n-1}} .
$$

Then for $n=0,1,2, \ldots$,

$$
\begin{gathered}
x_{2 n-2}=\frac{c(a e)^{n}}{\prod_{i=0}^{n-1}(e-i a)(c-i e)}, \\
x_{2 n-1}=\frac{b(b d)^{n}}{\prod_{i=0}^{n-1}(b-i d)(f-(i+1) b)}, \\
y_{2 n-2}=\frac{f(b d)^{n}}{\prod_{i=0}^{n-1}(b-i d)(f-i b)}, \\
y_{2 n-1}=\frac{e(a e)^{n}}{\prod_{i=0}^{n-1}(e-i a)(c-(i+1) e)} .
\end{gathered}
$$

Example 6. We consider an interesting numerical example for system (10) with the initial conditions $x_{-2}=0.18, x_{-1}=$ $-0.4, x_{0}=0.2, y_{-2}=0.03, y_{-1}=0.5$, and $y_{0}=0.26$. See Figure 1.

3. On the System: $x_{n+1}=x_{n-1} y_{n} /\left(x_{n-1}+y_{n-2}\right)$,

$$
y_{n+1}=x_{n} y_{n-1} /\left(y_{n-1}-x_{n-2}\right)
$$

In this section, we obtain the solutions form for the following system of two difference equations:

$$
x_{n+1}=\frac{x_{n-1} y_{n}}{x_{n-1}+y_{n-2}}, \quad y_{n+1}=\frac{x_{n} y_{n-1}}{y_{n-1}-x_{n-2}},
$$

with nonzero real numbers initial conditions $x_{-2}, x_{-1}, x_{0}, y_{-2}$, $y_{-1}$, and $y_{0}$ provided that $x_{-2} \neq y_{-1}$ and $x_{-1} \neq y_{0}$.

Theorem 7. Suppose that $\left\{x_{n}, y_{n}\right\}$ is a solution for system (23). Then for $n=0,1,2, \ldots$,

$$
\begin{aligned}
& x_{4 n-2} \\
& \quad=\frac{(a e)^{2 n}}{c^{n-1}(e-c)^{n} \prod_{i=0}^{n-1}(2 i a+e)((2 i+1) a+e)},
\end{aligned}
$$

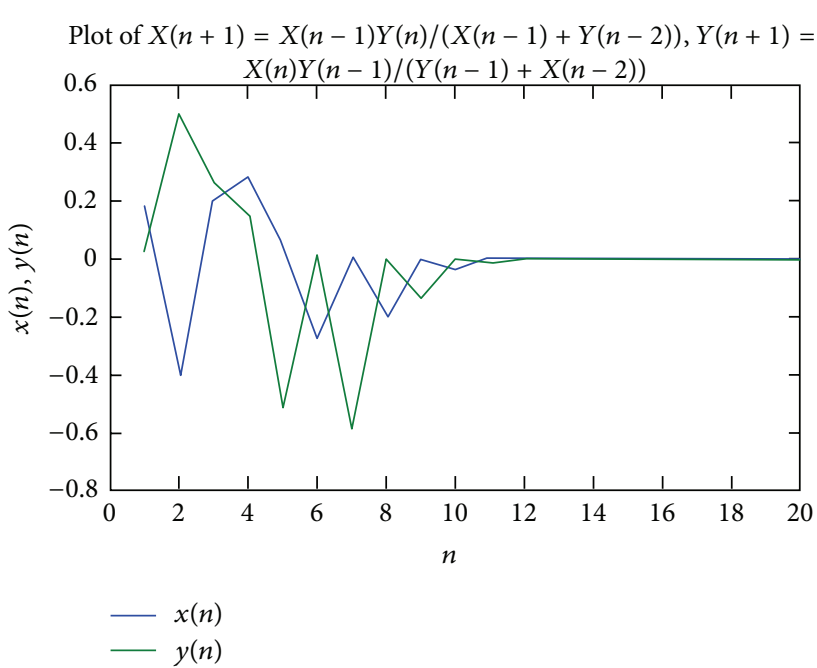

Figure 1

$$
\begin{aligned}
& x_{4 n-1} \\
& \quad=\frac{b^{n+1} d^{2 n}}{(d-b)^{n} \prod_{i=0}^{n-1}((2 i+1) b+f)((2 i+2) b+f)}, \\
& x_{4 n} \\
& \quad=\frac{a(a e)^{2 n}}{c^{n}(e-c)^{n} \prod_{i=0}^{n-1}((2 i+1) a+e)((2 i+2) a+e)}, \\
& x_{4 n+1} \\
& \quad=\frac{b^{n+1} d^{2 n+1}}{(b+f)(d-b)^{n} \prod_{i=0}^{n-1}((2 i+2) b+f)((2 i+3) b+f)},
\end{aligned}
$$

$y_{4 n-2}$

$$
=\frac{f b^{n} d^{2 n}}{(d-b)^{n} \prod_{i=0}^{n-1}((2 i) b+f)((2 i+1) b+f)},
$$

$y_{4 n-1}$

$$
=\frac{e(a e)^{2 n}}{c^{n}(e-c)^{n} \prod_{i=0}^{n-1}((2 i) a+e)((2 i+1) a+e)},
$$

$y_{4 n}$

$$
=\frac{b^{n} d^{2 n+1}}{(d-b)^{n} \prod_{i=0}^{n-1}((2 i+1) b+f)((2 i+2) b+f)},
$$

$y_{4 n+1}$

$$
=\frac{(a e)^{2 n+1}}{c^{n}(e-c)^{n+1} \prod_{i=0}^{n-1}((2 i+1) a+e)((2 i+2) a+e)} .
$$


Proof. For $n=0$ the result holds. Now suppose that $n>0$ and that our assumption holds for $n-1$. That is,

$x_{4 n-6}$

$$
=\frac{(a e)^{2 n-2}}{c^{n-2}(e-c)^{n-1} \prod_{i=0}^{n-2}(2 i a+e)((2 i+1) a+e)},
$$

$x_{4 n-5}$

$$
=\frac{b^{n} d^{2 n-2}}{(d-b)^{n-1} \prod_{i=0}^{n-2}((2 i+1) b+f)((2 i+2) b+f)},
$$

$x_{4 n-4}$

$$
=\frac{a(a e)^{2 n-2}}{c^{n-1}(e-c)^{n-1} \prod_{i=0}^{n-2}((2 i+1) a+e)((2 i+2) a+e)},
$$

$x_{4 n-3}$

$$
=\frac{b^{n} d^{2 n-1}}{(b+f)(d-b)^{n-1} \prod_{i=0}^{n-2}((2 i+2) b+f)((2 i+3) b+f)},
$$

$y_{4 n-6}$

$$
=\frac{f b^{n-1} d^{2 n-2}}{(d-b)^{n-1} \prod_{i=0}^{n-1}((2 i) b+f)((2 i+1) b+f)},
$$

$y_{4 n-5}$

$$
=\frac{e(a e)^{2 n-2}}{c^{n-1}(e-c)^{n-1} \prod_{i=0}^{n-2}((2 i) a+e)((2 i+1) a+e)},
$$

$y_{4 n-4}$

$$
=\frac{b^{n-1} d^{2 n-1}}{(d-b)^{n-1} \prod_{i=0}^{n-2}((2 i+1) b+f)((2 i+2) b+f)},
$$

$y_{4 n-3}$

$$
=\frac{(a e)^{2 n-1}}{c^{n-1}(e-c)^{n} \prod_{i=0}^{n-2}((2 i+1) a+e)((2 i+2) a+e)} .
$$

Now, it follows from system (23) that

$$
\begin{aligned}
& x_{4 n-2} \\
& =\frac{x_{4 n-4} y_{4 n-3}}{x_{4 n-4}+y_{4 n-5}} \\
& =\left(\frac{a(a e)^{2 n-2}}{c^{n-1}(e-c)^{n-1} \prod_{i=0}^{n-2}((2 i+1) a+e)((2 i+2) a+e)}\right) \\
& \quad \times\left(\frac{(a e)^{2 n-1}}{c^{n-1}(e-c)^{n} \prod_{i=0}^{n-2}((2 i+1) a+e)((2 i+2) a+e)}\right)
\end{aligned}
$$

$$
\begin{aligned}
& \times\left(\left(\frac{a(a e)^{2 n-2}}{c^{n-1}(e-c)^{n-1} \prod_{i=0}^{n-2}((2 i+1) a+e)((2 i+2) a+e)}\right)\right. \\
& \left.+\left(\frac{e(a e)^{2 n-2}}{c^{n-1}(e-c)^{n-1} \prod_{i=0}^{n-2}((2 i) a+e)((2 i+1) a+e)}\right)\right)^{-1} \\
& =\left(\frac{a(a e)^{2 n-1}}{c^{n-1}(e-c)^{n} \prod_{i=0}^{n-2}((2 i+1) a+e)((2 i+2) a+e)}\right) \\
& \times(a+(2 n-2) a+e)^{-1} \\
& =\frac{1}{((2 n-1) a+e)} \\
& \times \frac{a(a e)^{2 n-1}}{c^{n-1}(e-c)^{n} \prod_{i=0}^{n-2}((2 i+1) a+e)((2 i+2) a+e)} \\
& =\frac{(a e)^{2 n}}{c^{n-1}(e-c)^{n} \prod_{i=0}^{n-1}((2 i) a+e)((2 i+1) a+e)}, \\
& y_{4 n-2} \\
& =\frac{x_{4 n-3} y_{4 n-4}}{y_{4 n-4}-x_{4 n-5}} \\
& =\left(b ^ { n } d ^ { 2 n - 1 } \left((b+f)(d-b)^{n-1}\right.\right. \\
& \left.\left.\times \prod_{i=0}^{n-2}((2 i+2) b+f)((2 i+3) b+f)\right)^{-1}\right) \\
& \times\left(\frac{b^{n-1} d^{2 n-1}}{(d-b)^{n-1} \prod_{i=0}^{n-2}((2 i+1) b+f)((2 i+2) b+f)}\right) \\
& \times\left(\left(\frac{b^{n-1} d^{2 n-1}}{(d-b)^{n-1} \prod_{i=0}^{n-2}((2 i+1) b+f)((2 i+2) b+f)}\right)\right. \\
& \left.-\left(\frac{b^{n} d^{2 n-2}}{(d-b)^{n-1} \prod_{i=0}^{n-2}((2 i+1) b+f)((2 i+2) b+f)}\right)\right)^{-1} \\
& =\left(b ^ { n } d ^ { 2 n } \left((b+f)(d-b)^{n-1}\right.\right. \\
& \left.\left.\times \prod_{i=0}^{n-2}((2 i+2) b+f)((2 i+3) b+f)\right)^{-1}\right) \\
& \times(d-b)^{-1} \\
& =\frac{f b^{n} d^{2 n}}{(d-b)^{n} \prod_{i=0}^{n-1}((2 i) b+f)((2 i+1) b+f)} .
\end{aligned}
$$

Similarly one can prove the other relations. The proof is complete.

Lemma 8. Every positive solution of the equation $x_{n+1}=$ $x_{n-1} y_{n} /\left(x_{n-1}+y_{n-2}\right)$ is bounded, and $\lim _{n \rightarrow \infty} x_{n}=0$. 
The following theorems deal with the solutions form for the following systems, and their proofs will be omitted:

$$
\begin{aligned}
x_{n+1} & =\frac{x_{n-1} y_{n}}{y_{n-2}-x_{n-1}}, & y_{n+1} & =\frac{x_{n} y_{n-1}}{-y_{n-1}-x_{n-2}}, \\
x_{n+1} & =\frac{x_{n-1} y_{n}}{y_{n-2}-x_{n-1}}, & y_{n+1} & =\frac{x_{n} y_{n-1}}{y_{n-1}-x_{n-2}}, \\
x_{n+1} & =\frac{x_{n-1} y_{n}}{x_{n-1}+y_{n-2}}, & y_{n+1} & =\frac{x_{n} y_{n-1}}{-y_{n-1}-x_{n-2}} .
\end{aligned}
$$

Theorem 9. Assume that $\left\{x_{n}, y_{n}\right\}$ is a solution for system (27) with $x_{-2} \neq-y_{-1}$ and $x_{-1} \neq-y_{0}$. Then for $n=0,1,2, \ldots$,

$$
\begin{aligned}
& x_{4 n-2} \\
& \quad=\frac{(-1)^{n}(a e)^{2 n}}{c^{n-1}(e+c)^{n} \prod_{i=0}^{n-1}(e-2 i a)(e-(2 i+1) a)}, \\
& x_{4 n-1} \\
& \quad=\frac{(-1)^{n} b^{n+1} d^{2 n}}{(d+b)^{n} \prod_{i=0}^{n-1}((2 i+1) b-f)((2 i+2) b-f)}, \\
& \quad=\frac{(-1)^{n} a(a e)^{2 n}}{x^{n}(e+c)^{n} \prod_{i=0}^{n-1}(e-(2 i+1) a)(e-(2 i+2) a)}, \\
& x_{4 n+1} \\
& \quad=\frac{(-1)^{n+1} b^{n+1} d^{2 n+1}}{(b-f)(d+b)^{n} \prod_{i=0}^{n-1}((2 i+2) b-f)((2 i+3) b-f)},
\end{aligned}
$$

$y_{4 n-2}$

$$
=\frac{(-1)^{n} f b^{n} d^{2 n}}{(d+b)^{n} \prod_{i=0}^{n-1}((2 i) b-f)((2 i+1) b-f)},
$$

$y_{4 n-1}$

$$
=\frac{(-1)^{n} e(a e)^{2 n}}{c^{n}(e+c)^{n} \prod_{i=0}^{n-1}(e-(2 i) a)(e-(2 i+1) a)},
$$

$y_{4 n}$

$$
=\frac{(-1)^{n} b^{n} d^{2 n+1}}{(d+b)^{n} \prod_{i=0}^{n-1}((2 i+1) b-f)((2 i+2) b-f)},
$$

$y_{4 n+1}$

$$
=\frac{(-1)^{n+1}(a e)^{2 n+1}}{c^{n}(e+c)^{n+1} \prod_{i=0}^{n-1}(e-(2 i+1) a)(e-(2 i+2) a)} .
$$

Theorem 10. Assume that $\left\{x_{n}, y_{n}\right\}$ is a solution for system (28) with $x_{-2} \neq y_{-1}$ and $x_{-1} \neq y_{0}$. Then for $n=0,1,2, \ldots$,

$$
\begin{aligned}
& x_{4 n-2}=\frac{(-1)^{n}(a e)^{2 n}}{c^{n-1}(c-e)^{n} \prod_{i=0}^{n-1}(e-2 i a)(e-(2 i+1) a)}, \\
& x_{4 n-1}=\frac{(-1)^{n} b^{n+1} d^{2 n}}{(b-d)^{n} \prod_{i=0}^{n-1}(f-(2 i+1) b)(f-(2 i+2) b)}, \\
& x_{4 n} \\
& =\frac{(-1)^{n} a(a e)^{2 n}}{c^{n}(c-e)^{n} \prod_{i=0}^{n-1}(e-(2 i+1) a)(e-(2 i+2) a)}, \\
& x_{4 n+1} \\
& =\frac{(-1)^{n} b^{n+1} d^{2 n+1}}{(f-b)(b-d)^{n} \prod_{i=0}^{n-1}(f-(2 i+2) b)(f-(2 i+3) b)}, \\
& y_{4 n-2}=\frac{(-1)^{n} f b^{n} d^{2 n}}{(b-d)^{n} \prod_{i=0}^{n-1}(f-(2 i) b)(f-(2 i+1) b)}, \\
& y_{4 n-1}=\frac{(-1)^{n} e(a e)^{2 n}}{c^{n}(c-e)^{n} \prod_{i=0}^{n-1}(e-(2 i) a)(e-(2 i+1) a)}, \\
& y_{4 n}=\frac{(-1)^{n} b^{n} d^{2 n+1}}{(b-d)^{n} \prod_{i=0}^{n-1}(f-(2 i+1) b)(f-(2 i+2) b)}, \\
& y_{4 n+1} \\
& =\frac{(-1)^{n+1}(a e)^{2 n+1}}{c^{n}(c-e)^{n+1} \prod_{i=0}^{n-1}(e-(2 i+1) a)(e-(2 i+2) a)} .
\end{aligned}
$$

Theorem 11. The solution form for system (29) is given by

$$
\begin{aligned}
& x_{4 n-2}=\frac{(-1)^{n}(a e)^{2 n}}{c^{n-1}(c+e)^{n} \prod_{i=0}^{n-1}(e+2 i a)(e+(2 i+1) a)}, \\
& x_{4 n-1}=\frac{(-1)^{n} b^{n+1} d^{2 n}}{(b+d)^{n} \prod_{i=0}^{n-1}(f+(2 i+1) b)(f+(2 i+2) b)}, \\
& x_{4 n}=\frac{(-1)^{n} a(a e)^{2 n}}{c^{n}(c+e)^{n} \prod_{i=0}^{n-1}(e+(2 i+1) a)(e+(2 i+2) a)},
\end{aligned}
$$

$x_{4 n+1}$

$$
\begin{gathered}
=\frac{(-1)^{n} b^{n+1} d^{2 n+1}}{(f+b)(b+d)^{n} \prod_{i=0}^{n-1}(f+(2 i+2) b)(f+(2 i+3) b)}, \\
y_{4 n-2}=\frac{(-1)^{n} f b^{n} d^{2 n}}{(b+d)^{n} \prod_{i=0}^{n-1}(f+(2 i) b)(f+(2 i+1) b)}, \\
y_{4 n-1}=\frac{(-1)^{n} e(a e)^{2 n}}{c^{n}(c+e)^{n} \prod_{i=0}^{n-1}(e+(2 i) a)(e+(2 i+1) a)}, \\
y_{4 n}=\frac{(-1)^{n} b^{n} d^{2 n+1}}{(b+d)^{n} \prod_{i=0}^{n-1}(f+(2 i+1) b)(f+(2 i+2) b)},
\end{gathered}
$$




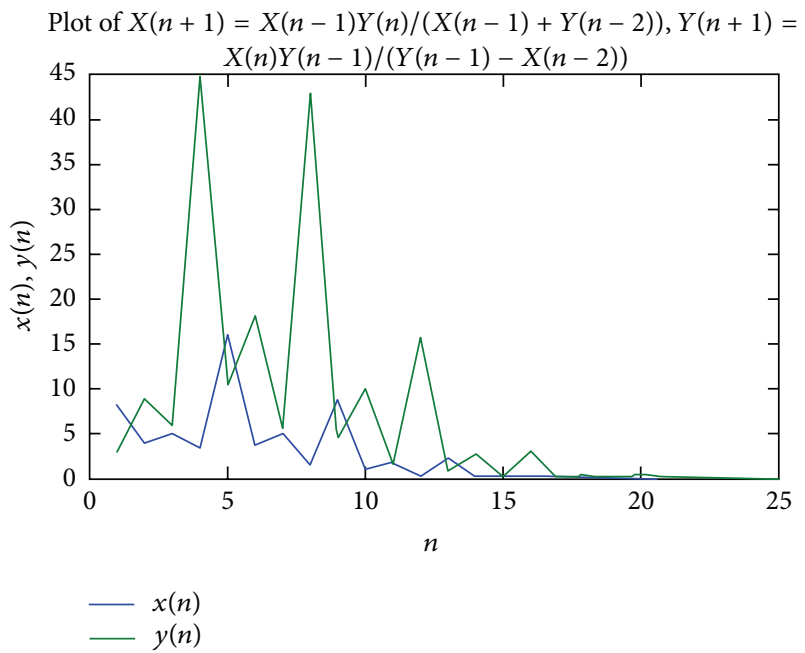

FIGURE 2

$y_{4 n+1}$

$$
=\frac{(-1)^{n+1}(a e)^{2 n+1}}{c^{n}(c+e)^{n+1} \prod_{i=0}^{n-1}(e+(2 i+1) a)(e+(2 i+2) a)},
$$

where $x_{-2} \neq-y_{-1}$ and $x_{-1} \neq-y_{0}$.

Example 12. Consider system (23) with the initial conditions $x_{-2}=8, x_{-1}=4, x_{0}=5, y_{-2}=3, y_{-1}=9$, and $y_{0}=6$. See Figure 2 .

4. On the System: $x_{n+1}=x_{n-1} y_{n} /\left(x_{n-1}-y_{n-2}\right)$,

$$
y_{n+1}=x_{n} y_{n-1} /\left(y_{n-1}+x_{n-2}\right)
$$

In this section, we present the solutions form for the following system:

$$
x_{n+1}=\frac{x_{n-1} y_{n}}{x_{n-1}-y_{n-2}}, \quad y_{n+1}=\frac{x_{n} y_{n-1}}{y_{n-1}+x_{n-2}},
$$

with nonzero real numbers initial conditions where $x_{-1} \neq y_{-2}$ and $x_{0} \neq y_{-1}$.

The following theorems can be proved similarly to those in Sections 2 and 3.

Theorem 13. Suppose that $\left\{x_{n}, y_{n}\right\}$ is a solution for system (33). Assume that $x_{-2}, x_{-1}, x_{0}, y_{-2}, y_{-1}$, and $y_{0}$ are arbitrary nonzero real numbers. Then

$$
\begin{gathered}
x_{4 n-2}=\frac{c a^{2 n} e^{n}}{(a-e)^{n} \prod_{i=0}^{n-1}(2 i e+c)((2 i+1) e+c)}, \\
x_{4 n-1}=\frac{b^{2 n+1} d^{2 n}}{f^{n}(b-f)^{n} \prod_{i=0}^{n-1}((2 i) d+b)((2 i+1) d+b)},
\end{gathered}
$$

$$
\begin{gathered}
x_{4 n}=\frac{a^{2 n+1} e^{n}}{(a-e)^{n} \prod_{i=0}^{n-1}((2 i+1) e+c)((2 i+2) e+c)}, \\
x_{4 n+1}=\frac{(b d)^{2 n+1}}{f^{n}(b-f)^{n} \prod_{i=0}^{n-1}((2 i+1) d+b)((2 i+2) d+b)}, \\
y_{4 n-2}=\frac{(b d)^{2 n}}{f^{n-1}(b-f)^{n} \prod_{i=0}^{n-1}((2 i) d+b)((2 i+1) d+b)}, \\
y_{4 n-1}=\frac{a^{2 n} e^{n+1}}{(a-e)^{n} \prod_{i=0}^{n-1}((2 i+1) e+c)((2 i+2) e+c)}, \\
y_{4 n}=\frac{b^{2 n} d^{2 n+1}}{f^{n}(b-f)^{n} \prod_{i=0}^{n-1}((2 i+1) d+b)((2 i+2) d+b)}, \\
y_{4 n+1}=\frac{a^{2 n+1} e^{n+1}}{(c+e)(a-e)^{n} \prod_{i=0}^{n-1}((2 i+2) e+c)((2 i+3) e+c)} .
\end{gathered}
$$

Lemma 14. Every positive solution of the equation $y_{n+1}=$ $x_{n} y_{n-1} /\left(y_{n-1}+x_{n-2}\right)$ is bounded and $\lim _{n \rightarrow \infty} y_{n}=0$.

Theorem 15. Let $\left\{x_{n}, y_{n}\right\}$ be a solution for the system

$$
x_{n+1}=\frac{x_{n-1} y_{n}}{-x_{n-1}-y_{n-2}}, \quad y_{n+1}=\frac{x_{n} y_{n-1}}{x_{n-2}-y_{n-1}},
$$

with $x_{-1} \neq-y_{-2}$ and $x_{0} \neq-y_{-1}$. Then for $n=0,1,2, \ldots$,

$$
\begin{aligned}
& x_{4 n-2}=\frac{(-1)^{n} c a^{2 n} e^{n}}{(a+e)^{n} \prod_{i=0}^{n-1}(c-2 i e)(c-(2 i+1) e)}, \\
& x_{4 n-1}=\frac{(-1)^{n} b^{2 n+1} d^{2 n}}{f^{n}(b+f)^{n} \prod_{i=0}^{n-1}(b-(2 i) d)(b-(2 i+1) d)}, \\
& x_{4 n}=\frac{(-1)^{n} a^{2 n+1} e^{n}}{(a+e)^{n} \prod_{i=0}^{n-1}(c-(2 i+1) e)(c-(2 i+2) e)}, \\
& x_{4 n+1}=\frac{(-1)^{n+1}(b d)^{2 n+1}}{f^{n}(b+f)^{n} \prod_{i=0}^{n-1}(b-(2 i+1) d)(b-(2 i+2) d)}, \\
& y_{4 n-2}=\frac{(-1)^{n}(b d)^{2 n}}{f^{n-1}(b+f)^{n} \prod_{i=0}^{n-1}(b-(2 i) d)(b-(2 i+1) d)}, \\
& y_{4 n-1}=\frac{(-1)^{n} a^{2 n} e^{n+1}}{(a+e)^{n} \prod_{i=0}^{n-1}(c-(2 i+1) e)(c-(2 i+2) e)}, \\
& y_{4 n}=\frac{(-1)^{n} b^{2 n} d^{2 n+1}}{f^{n}(b+f)^{n} \prod_{i=0}^{n-1}(b-(2 i+1) d)(b-(2 i+2) d)}, \\
& y_{4 n+1}=\frac{(-1)^{n} a^{2 n+1} e^{n+1}}{(c-e)(a+e)^{n} \prod_{i=0}^{n-1}(c-(2 i+2) e)(c-(2 i+3) e)} .
\end{aligned}
$$

Theorem 16. The solution form for the following system

$$
x_{n+1}=\frac{x_{n-1} y_{n}}{-x_{n-1}-y_{n-2}}, \quad y_{n+1}=\frac{x_{n} y_{n-1}}{x_{n-2}+y_{n-1}},
$$

with $x_{-1} \neq-y_{-2}$ and $x_{0} \neq-y_{-1}$ is given by 


$$
\begin{gathered}
x_{4 n-2}=\frac{(-1)^{n} c a^{2 n} e^{n}}{(a+e)^{n} \prod_{i=0}^{n-1}(c+2 i e)(c+(2 i+1) e)}, \\
x_{4 n-1}=\frac{(-1)^{n} b^{2 n+1} d^{2 n}}{f^{n}(b+f)^{n} \prod_{i=0}^{n-1}(b+(2 i) d)(b+(2 i+1) d)}, \\
x_{4 n}=\frac{(-1)^{n} a^{2 n+1} e^{n}}{(a+e)^{n} \prod_{i=0}^{n-1}(c+(2 i+1) e)(c+(2 i+2) e)}, \\
x_{4 n+1}=\frac{(-1)^{n+1}(b d)^{2 n+1}}{f^{n}(b+f)^{n} \prod_{i=0}^{n-1}(b+(2 i+1) d)(b+(2 i+2) d)}, \\
y_{4 n-1}=\frac{(-1)^{n}(b d)^{2 n}}{f^{n-1}(b+f)^{n} \prod_{i=0}^{n-1}(b+(2 i) d)(b+(2 i+1) d)}, \\
y_{4 n}=\frac{(-1)^{n} a^{2 n} e^{n+1}}{f^{n}(b+f)^{n} \prod_{i=0}^{n-1}(b+(2 i+1) d)(b+(2 i+2) d)}, \\
=\frac{(-1)^{n} a^{2 n+1} e^{n+1}}{(c+e)(a+e)^{n} \prod_{i=0}^{n-1}(c+(2 i+2) e)(c+(2 i+3) e)}
\end{gathered}
$$

Theorem 17. The following system

$$
x_{n+1}=\frac{x_{n-1} y_{n}}{x_{n-1}-y_{n-2}}, \quad y_{n+1}=\frac{x_{n} y_{n-1}}{x_{n-2}-y_{n-1}}
$$

has a solution form given by the following relations:

$$
\begin{gathered}
x_{4 n-2}=\frac{c a^{2 n} e^{n}}{(a-e)^{n} \prod_{i=0}^{n-1}(c-2 i e)(c-(2 i+1) e)}, \\
x_{4 n-1}=\frac{b^{2 n+1} d^{2 n}}{f^{n}(b-f)^{n} \prod_{i=0}^{n-1}(b-(2 i) d)(b-(2 i+1) d)}, \\
x_{4 n}=\frac{a^{2 n+1} e^{n}}{(a-e)^{n} \prod_{i=0}^{n-1}(c-(2 i+1) e)(c-(2 i+2) e)}, \\
x_{4 n+1}=\frac{(b d)^{2 n+1}}{f^{n}(b-f)^{n} \prod_{i=0}^{n-1}(b-(2 i+1) d)(b-(2 i+2) d)}, \\
y_{4 n-1}=\frac{(b d)^{2 n}}{f^{n-1}(b-f)^{n} \prod_{i=0}^{n-1}(b-(2 i) d)(b-(2 i+1) d)}, \\
y_{4 n}=\frac{a^{2 n} e^{n+1}}{f^{n}(b-f)^{n} \prod_{i=0}^{n-1}(b-(2 i+1) d)(b-(2 i+2) d)}, \\
(c-e)(a-e)^{n} \prod_{i=0}^{n-1}(c-(2 i+2) e)(c-(2 i+3) e)
\end{gathered},
$$

where $x_{-1} \neq y_{-2}$ and $x_{0} \neq y_{-1}$.

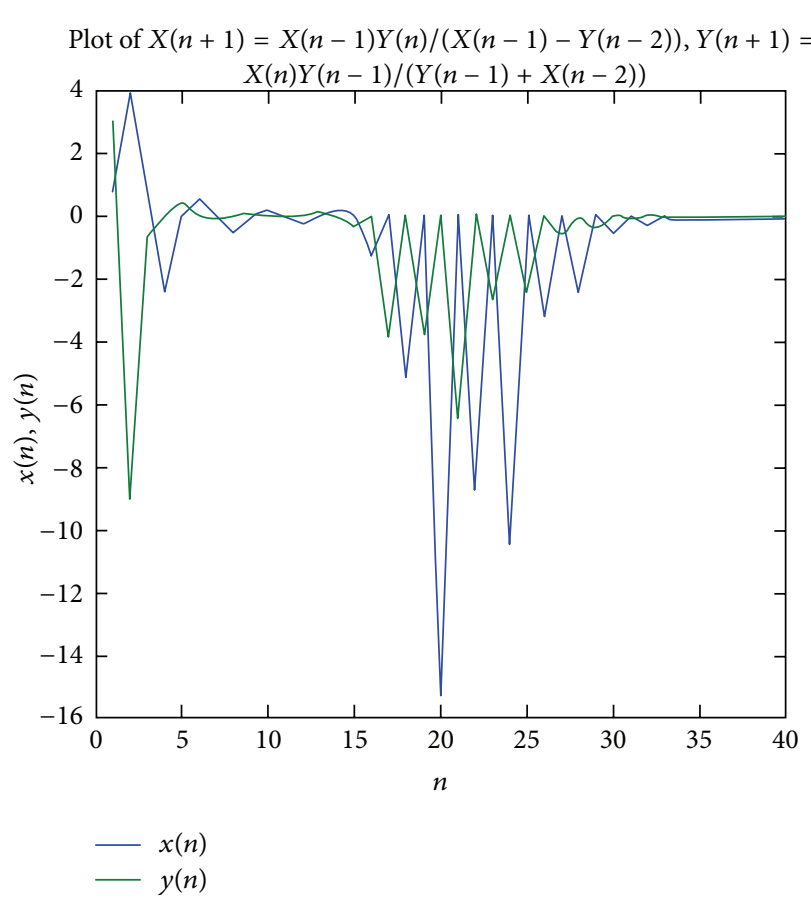

Figure 3

Example 18. Consider system (33) with the initial values $x_{-2}=0.8, x_{-1}=4, x_{0}=0.15, y_{-2}=3, y_{-1}=-9$, and $y_{0}=-0.6$. See Figure 3 .

\section{Other Systems}

In this section, we give the solutions form for the following systems of difference equations:

$$
\begin{array}{cc}
x_{n+1}=\frac{x_{n-1} y_{n}}{x_{n-1}-y_{n-2}}, & y_{n+1}=\frac{x_{n} y_{n-1}}{y_{n-1}-x_{n-2}}, \\
x_{n+1}=\frac{x_{n-1} y_{n}}{-x_{n-1}-y_{n-2}}, & y_{n+1}=\frac{x_{n} y_{n-1}}{y_{n-1}-x_{n-2}}, \\
x_{n+1}=\frac{x_{n-1} y_{n}}{x_{n-1}-y_{n-2}}, & y_{n+1}=\frac{x_{n} y_{n-1}}{-y_{n-1}-x_{n-2}}, \\
x_{n+1}=\frac{x_{n-1} y_{n}}{-x_{n-1}-y_{n-2}}, & y_{n+1}=\frac{x_{n} y_{n-1}}{-y_{n-1}-x_{n-2}},
\end{array}
$$

with nonzero real numbers initial conditions.

Theorem 19. Let $\left\{x_{n}, y_{n}\right\}$ be a solution for system (41) with $x_{-2} \neq y_{-1} \neq x_{0}$ and $y_{-2} \neq x_{-1} \neq y_{0}$. Then

$$
\begin{aligned}
& x_{4 n-2}=\frac{a^{2 n} e^{n}}{c^{n-1}[(a-e)(e-c)]^{n}}, \\
& x_{4 n-1}=\frac{b^{n+1} d^{2 n}}{[f(b-d)(f-b)]^{n}},
\end{aligned}
$$




$$
\begin{gathered}
x_{4 n}=\frac{a^{2 n+1} e^{n}}{[c(a-e)(e-c)]^{n}}, \\
x_{4 n+1}=\frac{-d^{2 n+1} b^{n+1}}{(f-b)[f(b-d)(f-b)]^{n}}, \\
y_{4 n-2}=\frac{f b^{n} d^{2 n}}{[f(b-d)(f-b)]^{n}}, \\
y_{4 n-1}=\frac{a^{2 n} e^{n+1}}{[c(a-e)(e-c)]^{n}}, \\
y_{4 n}=\frac{b^{n} d^{2 n+1}}{[f(b-d)(f-b)]^{n}}, \\
y_{4 n+1}=\frac{a^{2 n+1} e^{n+1}}{(e-c)[c(a-e)(e-c)]^{n}} .
\end{gathered}
$$

Theorem 20. Suppose that $\left\{x_{n}, y_{n}\right\}$ is a solution for system (42) with $x_{-2} \neq y_{-1}, y_{-1} \neq-x_{0}, y_{-2} \neq-x_{-1}$, and $x_{-1} \neq y_{0}$. Then

$$
\begin{gathered}
x_{4 n-2}=\frac{a^{2 n} e^{n}}{c^{n-1}[(a+e)(c-e)]^{n}}, \\
x_{4 n-1}=\frac{b^{n+1} d^{2 n}}{[f(b-d)(f+b)]^{n}}, \\
x_{4 n}=\frac{a^{2 n+1} e^{n}}{[c(a+e)(c-e)]^{n}}, \\
x_{4 n+1}=\frac{(f+b)[f(b-d)(f+b)]^{n}}{\left(f+1 b^{n+1}\right.} \\
y_{4 n-2}=\frac{f b^{n} d^{2 n}}{[f(b-d)(f+b)]^{n}}, \\
y_{4 n-1}=\frac{a^{2 n} e^{n+1}}{[c(a+e)(c-e)]^{n}}, \\
y_{4 n}=\frac{b^{n} d^{2 n+1}}{[f(b-d)(f+b)]^{n}}, \\
y_{4 n+1}=\frac{-a^{2 n+1} e^{n+1}}{(c-e)[c(a+e)(c-e)]^{n}} .
\end{gathered}
$$

Theorem 21. The solution for system (43) is given by the following formula; for $n=0,1,2, \ldots$;

$$
\begin{aligned}
x_{4 n-2} & =\frac{a^{2 n} e^{n}}{c^{n-1}[(e-a)(c+e)]^{n}}, \\
x_{4 n-1} & =\frac{b^{n+1} d^{2 n}}{[f(b+d)(f-b)]^{n}}, \\
x_{4 n} & =\frac{a^{2 n+1} e^{n}}{[c(e-a)(c+e)]^{n}},
\end{aligned}
$$

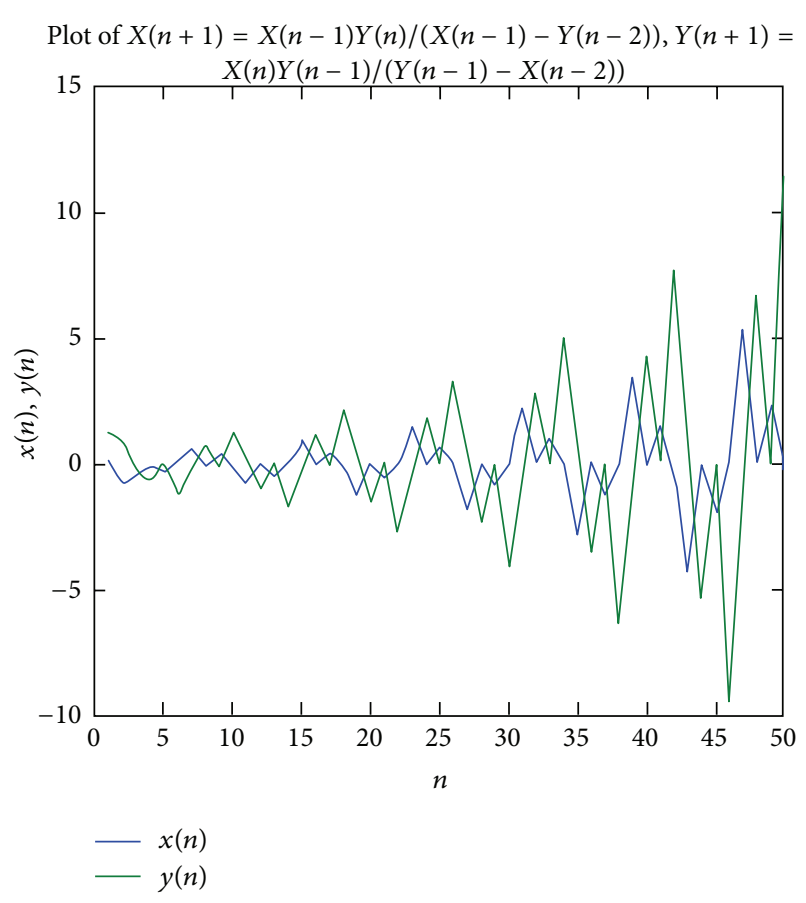

Figure 4

$$
\begin{gathered}
x_{4 n+1}=\frac{-d^{2 n+1} b^{n+1}}{(f-b)[f(b+d)(f-b)]^{n}}, \\
y_{4 n-2}=\frac{f b^{n} d^{2 n}}{[f(b+d)(f-b)]^{n}}, \\
y_{4 n-1}=\frac{a^{2 n} e^{n+1}}{[c(e-a)(c+e)]^{n}}, \\
y_{4 n}=\frac{b^{n} d^{2 n+1}}{[f(b+d)(f-b)]^{n}}, \\
y_{4 n+1}=\frac{-a^{2 n+1} e^{n+1}}{(c+e)[c(e-a)(c+e)]^{n}},
\end{gathered}
$$

where $x_{-2} \neq-y_{-1}, y_{-1} \neq x_{0}, y_{-2} \neq x_{-1}$, and $x_{-1} \neq-y_{0}$.

Theorem 22. If $\left\{x_{n}, y_{n}\right\}$ is a solution for system (44) with $x_{-2} \neq-y_{-1}, y_{-1} \neq-x_{0}, y_{-2} \neq-x_{-1}$, and $x_{-1} \neq-y_{0}$, then

$$
\begin{gathered}
x_{4 n-2}=\frac{a^{2 n} e^{n}}{c^{n-1}[(e+a)(c+e)]^{n}}, \\
x_{4 n-1}=\frac{b^{n+1} d^{2 n}}{[f(b+d)(f+b)]^{n}}, \\
x_{4 n}=\frac{a^{2 n+1} e^{n}}{[c(e+a)(c+e)]^{n}}, \\
x_{4 n+1}=\frac{-d^{2 n+1} b^{n+1}}{(f+b)[f(b+d)(f+b)]^{n}},
\end{gathered}
$$




$$
\begin{gathered}
y_{4 n-2}=\frac{f b^{n} d^{2 n}}{[f(b+d)(f+b)]^{n}}, \\
y_{4 n-1}=\frac{a^{2 n} e^{n+1}}{[c(e+a)(c+e)]^{n}}, \\
y_{4 n}=\frac{b^{n} d^{2 n+1}}{[f(b+d)(f+b)]^{n}}, \\
y_{4 n+1}=\frac{-a^{2 n+1} e^{n+1}}{(c+e)[c(e+a)(c+e)]^{n}} .
\end{gathered}
$$

Example 23. Figure 4 shows the behavior of the solution for system (41) with the initial conditions $x_{-2}=0.18, x_{-1}=-0.7$, $x_{0}=-0.5, y_{-2}=1.3, y_{-1}=0.9$, and $y_{0}=-0.26$.

\section{References}

[1] R. P. Agarwal, Difference Equations and Inequalities, Marcel Dekker, New York, NY, USA, 2nd edition, 2000.

[2] R. P. Agarwal and E. M. Elsayed, "On the solution of fourthorder rational recursive sequence," Advanced Studies in Contemporary Mathematics, vol. 20, no. 4, pp. 525-545, 2010.

[3] E. M. Elabbasy, H. El-Metwally, and E. M. Elsayed, "On the solutions of a class of difference equations systems," Demonstratio Mathematica, vol. 41, no. 1, pp. 109-122, 2008.

[4] H. El-Metwally, "Qualitative properties of some higher order difference equations," Computers \& Mathematics with Applications, vol. 58, no. 4, pp. 686-692, 2009.

[5] A. Y. Özban, "On the positive solutions of the system of rational difference equations $x_{n+1}=1 / y_{n-k}, y_{n+1}=y_{n} / x_{n-m} y_{n-m-k}$," Journal of Mathematical Analysis and Applications, vol. 323, no. 1, pp. 26-32, 2006.

[6] C. Wang, S. Wang, and X. Yan, "Global asymptotic stability of 3-species mutualism models with diffusion and delay effects," Discrete Dynamics in Nature and Society, vol. 2009, Article ID 317298, 20 pages, 2009.

[7] E. M. E. Zayed and M. A. El-Moneam, "On the rational recursive sequence $x_{n+1}=a x_{n}-b x_{n} /\left(c x_{n}-d x_{n-k}\right)$," Communications on Applied Nonlinear Analysis, vol. 15, no. 2, pp. 47-57, 2008.

[8] C. Cinar, I. Yalçinkaya, and R. Karatas, "On the positive solutions of the difference equation system $x_{n+1}=m / y_{n}, y_{n+1}=$ $p y_{n} / x_{n-1} y_{n-1}$," Journal of Institute of Mathematics and Computer Sciences, vol. 18, pp. 135-136, 2005.

[9] A. Y. Özban, "On the system of rational difference equations $x_{n+1}=a / y_{n-3}, y_{n+1}=b y_{n-3} / x_{n-q} y_{n-q}$, , Applied Mathematics and Computation, vol. 188, no. 1, pp. 833-837, 2007.

[10] A. S. Kurbanll, C. Çinar, and I. Yalçinkaya, "On the behavior of positive solutions of the system of rational difference equations," Mathematical and Computer Modelling, vol. 53, no. 5-6, pp. 1261-1267, 2011.

[11] N. Touafek and E. M. Elsayed, "On the periodicity of some systems of nonlinear difference equations," Bulletin Mathématiques de la Société des Sciences, vol. 55(103), no. 2, pp. 217-224, 2012.

[12] I. Yalcinkaya, "On the global asymptotic stability of a secondorder system of difference equations," Discrete Dynamics in Nature and Society, vol. 2008, Article ID 860152, 12 pages, 2008.

[13] X. Yang, "On the system of rational difference equations $x_{n}=$ $A+y_{n-1} / x_{n-p} y_{n-q}, A+x_{n-1} / x_{n-r} y_{n-s}$," Journal of Mathematical Analysis and Applications, vol. 307, no. 1, pp. 305-311, 2005.
[14] D. Clark and M. R. S. Kulenović, "A coupled system of rational difference equations," Computers \& Mathematics with Applications, vol. 43, no. 6-7, pp. 849-867, 2002.

[15] D. Clark, M. R. S. Kulenović, and J. F. Selgrade, "Global asymptotic behavior of a two-dimensional difference equation modelling competition," Nonlinear Analysis. Theory, Methods \& Applications, vol. 52, no. 7, pp. 1765-1776, 2003.

[16] E. Camouzis and G. Papaschinopoulos, "Global asymptotic behavior of positive solutions on the system of rational difference equations," Applied Mathematics Letters, vol. 17, no. 6, pp. 733-737, 2004. 


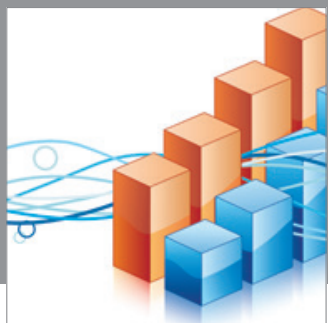

Advances in

Operations Research

mansans

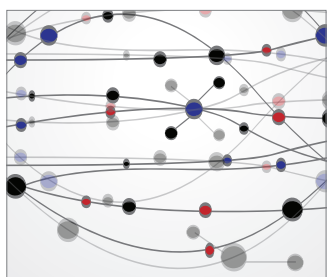

The Scientific World Journal
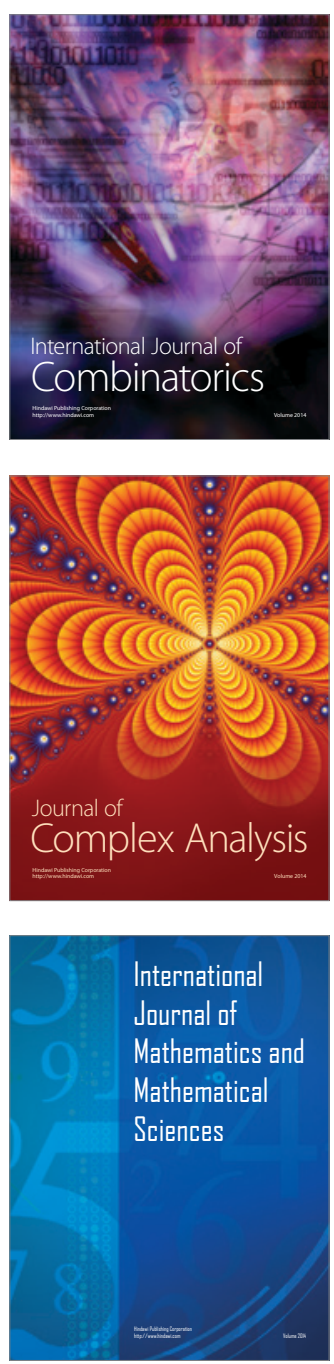
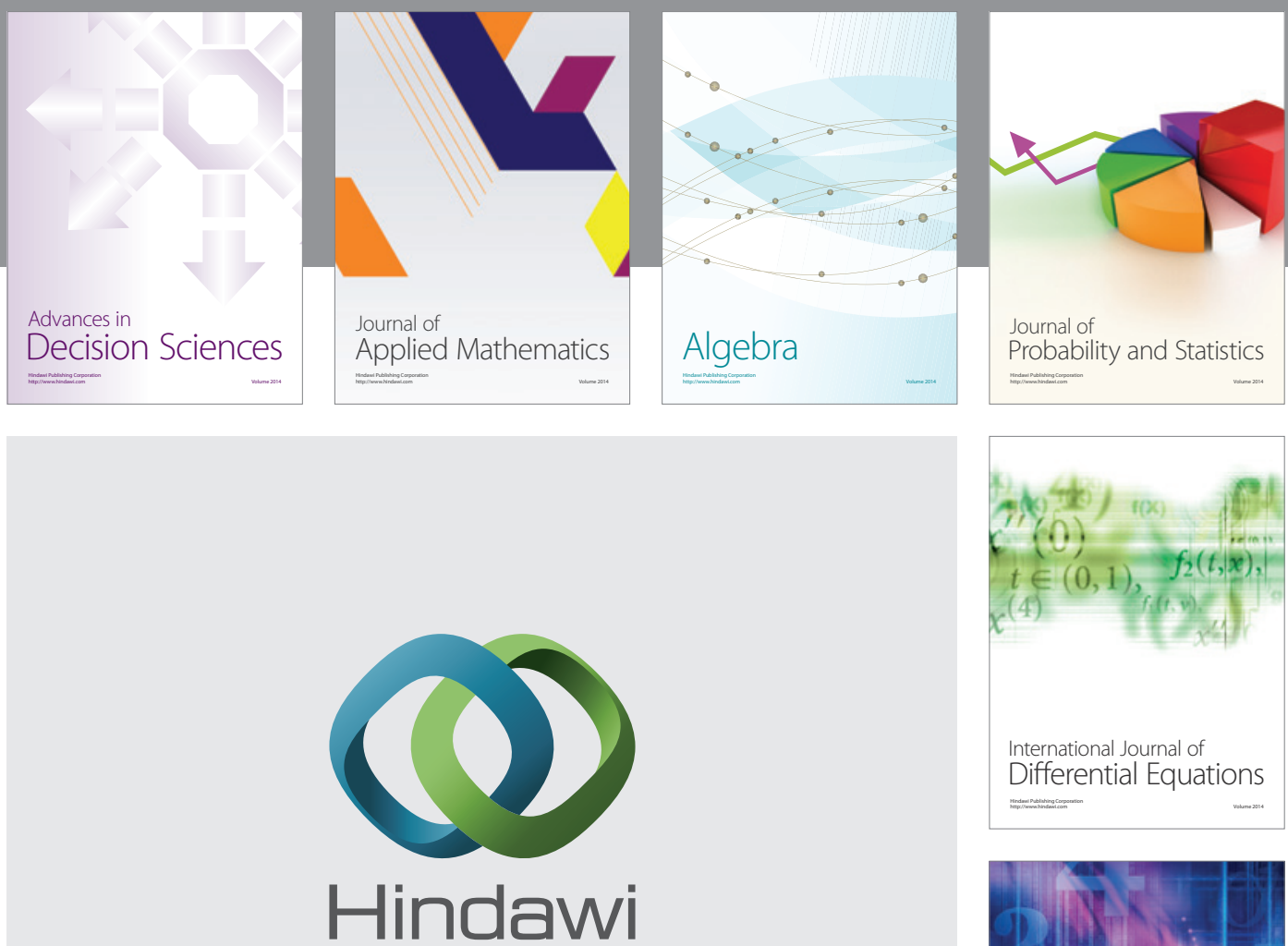

Submit your manuscripts at http://www.hindawi.com
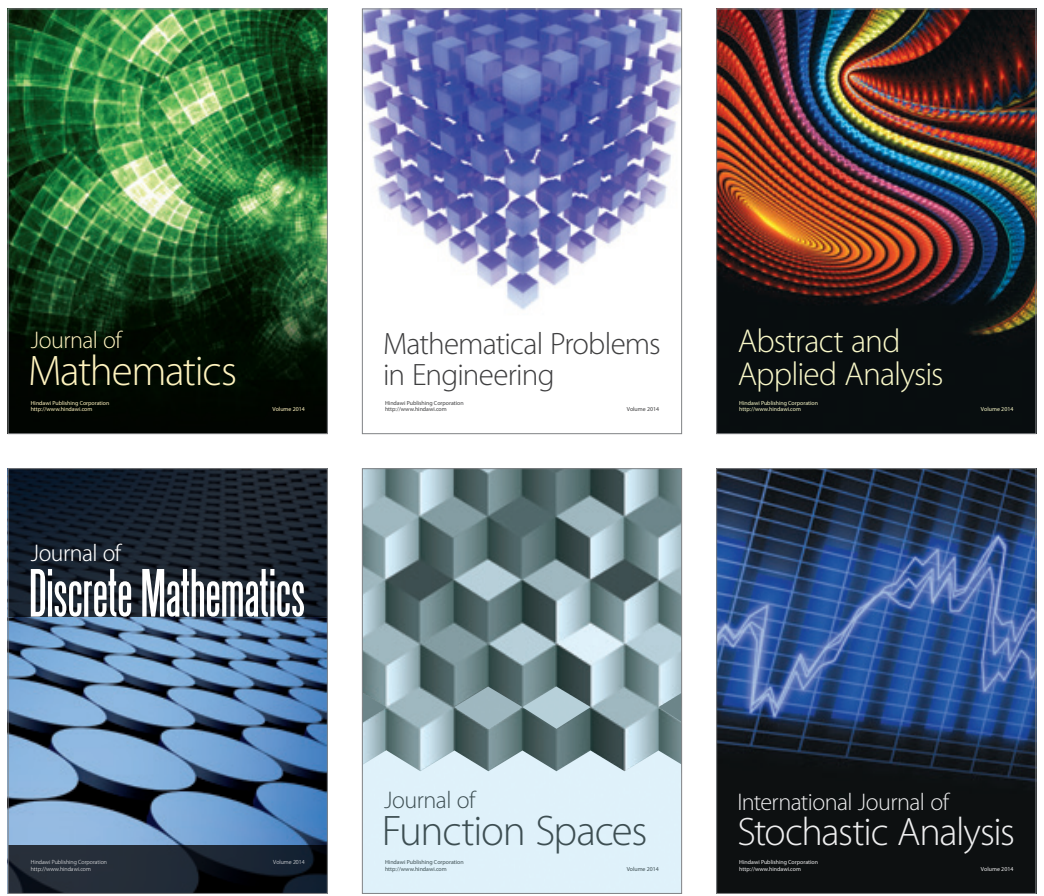

Journal of

Function Spaces

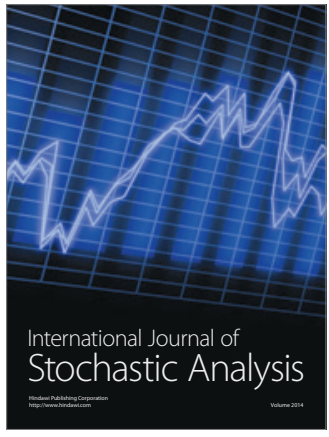

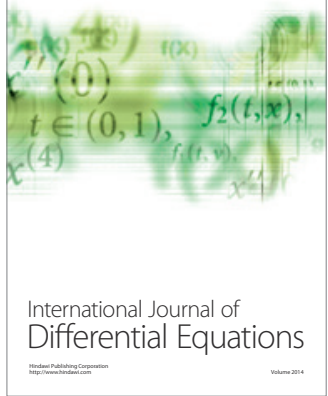
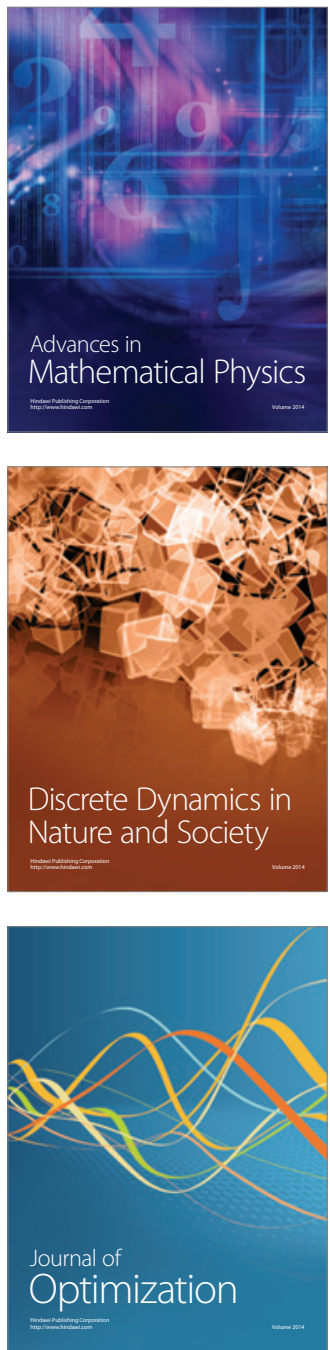